www.nature.com/pj

\title{
The static structure of polyrotaxane in solution investigated by contrast variation small-angle neutron scattering
}

\author{
Hitoshi Endo ${ }^{1,3}$, Koichi Mayumi ${ }^{2}$, Noboru Osaka ${ }^{1,4}$, Kohzo Ito $^{2}$ and Mitsuhiro Shibayama ${ }^{1}$ \\ Polyrotaxane (PR) possesses a supramolecular structure in which cyclic molecules are threaded into an axial polymer. In this \\ study, the static structure of PR dissolved in a good solvent was investigated using contrast variation small-angle neutron \\ scattering. The conformation of the axial linear polymer and the alignment of cyclic molecules within the axial polymer were \\ evaluated quantitatively with the help of a detailed derivation of scattering theory. The decomposed partial scattering functions \\ of the cyclic molecules and the axial polymer and the cross-correlation between cyclic molecules and an axial polymer strongly \\ supported the idea that the alignment of cyclic molecules threaded on the axial polymer is random. On the basis of experimental \\ observation, the entropic origin of the stiffening of PR due to the array of cyclic molecules is discussed. \\ Polymer Journal (2011) 43, 155-163; doi:10.1038/pj.2010.124; published online 8 December 2010
}

Keywords: contrast variation; partial scattering function; polymer solution; polyrotaxane; small-angle neutron scattering; supramolecule

\section{INTRODUCTION}

The investigation of polymer solutions has long retained a fundamental place in the research of polymers. ${ }^{1}$ Because polymers cannot exist in a gaseous state, a simple experimental method for the characterization of a single polymer chain must be performed with dilute polymer solutions, in which the polymer molecules are well separated. Theoretically, the mean-square end-to-end distances, viscosity and dynamics of a single polymer chain have been intensively studied. $^{2,3}$ The first statistical mechanical approach for determining the configuration of a polymer in solution was developed by Kuhn; ${ }^{4}$ Flory then introduced the excluded-volume effect for the description of real polymer chains in good solvents. ${ }^{5}$ Some time later, the theory for the dilute solution behavior of flexible polymers was almost complete. ${ }^{1}$ However, the problems of stiff or semiflexible polymers still remained. These problems were solved by Yamakawa who proposed the helical worm-like chain model to describe the equilibrium conformational and dynamic properties of all kinds of real polymers across a large breadth of length scales; that is, the theory can bridge the gap between atomic-level models and continuum theories. ${ }^{3,6}$

The field of supramolecular chemistry has developed rapidly in recent times. ${ }^{7}$ Supramolecular species are held together and organize themselves by means of non-covalent binding interactions, such as intermolecular forces and electrostatic or hydrogen bonding. Polyrotaxane (PR), which is a topological copolymer composed of cyclic molecules threaded into a linear polymer chain, is one of the most promising supramolecules. ${ }^{8}$ The cyclic molecules in PR are mechanically interlocked by capping the axial chain with bulky end groups, and their degrees of freedom involve slide, rotation and transfer because of the diffusion of monomer units of the axial polymer. These unique kinetic properties have been used for the development of novel functional materials. ${ }^{9}$ For example, slide-ring gel has been produced by chemically crosslinking several cyclic molecules on different axial polymers. ${ }^{10}$ The properties of slide-ring gel are quite unique; for example, high extensibility, high swelling properties and high resistance to external stress. ${ }^{11}$

Unlike conventional covalent macromolecules, the physical properties of PR have just begun to be studied and are not yet well understood. A few small-angle neutron scattering (SANS) investigations have been conducted on dilute and semidilute solutions of PR. ${ }^{12-16}$ It has been found that the persistent length of PR is much longer than that of a flexible polymer with the same contour length, and the conformation of PR is much affected by the inclusion ratio of the cyclic molecules ${ }^{13}$ and the concentration of PR itself. ${ }^{15,16}$ Because PR possesses a binary-component supramolecular structure, a direct observation of the individual structures and dynamics of the cyclic

\footnotetext{
${ }^{1}$ Neutron Science Laboratory, Institute for Solid State Physics, The University of Tokyo, Ibaraki, Japan and ${ }^{2}$ Graduate School of Frontier Sciences, The University of Tokyo, Chiba, Japan

${ }^{3}$ Current address: Neutron Biophysics Group, Quantum Beam Science Directorate, Japan Atomic Energy Agency, 2-4 Shirane, Shirakata, Tokai-Mura, Ibaraki 319-1195, Japan. ${ }^{4}$ Current address: Department of Organic and Polymer Materials Chemistry, Tokyo University of Agriculture and Technology, 2-24-16 Nakamachi, Koganei, Tokyo 184-8588, Japan. Correspondence: Dr H Endo, Neutron Biophysics Group, Quantum Beam Science Directorate, Japan Atomic Energy Agency, 2-4 Shirane, Shirakata, Tokai-Mura, Ibaraki 319-1195, Japan. E-mail: endo.hitoshi@jaea.go.jp or

K Mayumi, Graduate School of Frontier Science, The University of Tokyo, 5-1-5 Kashiwanoha, Kashiwa, Chiba 277-8561, Japan.

E-mail: kmayumi@molle.k.u-tokyo.ac.jp
}

Received 15 May 2010; revised 21 October 2010; accepted 22 October 2010; published online 8 December 2010 


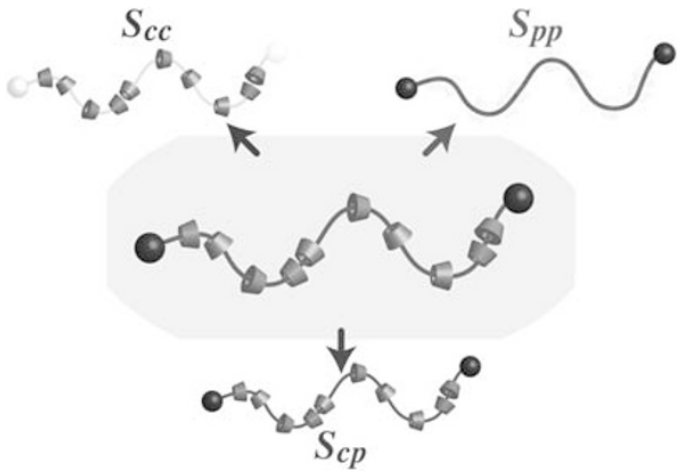

Figure 1 Schematic picture of the partial scattering functions in polyrotaxane. $S_{\mathrm{CC}}$ and $S_{\mathrm{PP}}$ reflect self-correlation of cyclic molecules and the axial polymer, respectively. $S_{\mathrm{CP}}$ represents cross-correlation between cyclic molecules and the axial polymer.

molecules and the axial polymer in PR has been difficult with conventional experimental techniques.

The aim of this study was to elucidate the detailed static structure of PR by means of contrast variation SANS (CV-SANS). Using neutrons as a probe, the contrast variation technique can be feasible on the basis of hydrogen/deuterium replacement to tune the visibility of different components in the system. By applying CV-SANS, the measured scattering intensities with different scattering contrasts can be decomposed into the partial scattering functions of each component, and detailed analyses of the partial scattering functions lead to fine structural understanding of the components. Regarding the observable partial scattering function in PR, two self-correlation functions for cyclic molecules and an axial polymer, as well as one cross-correlation function between cyclic molecules and the axial polymer, must be considered. In Figure 1, a schematic picture of the partial scattering functions of PR is depicted. Before this paper, a short article was published to introduce this study briefly. ${ }^{17}$ In this paper, we first derive the form factor for cyclic molecules threaded into the axial polymer by taking account of the one-dimensional distribution of cyclic molecules on the axis. At the same time, the form factor of the axial polymer and the cross-correlation between cyclic molecules and the axis could be derived in a manner similar to the derivation of the form factor for cyclic molecules. The total evaluation of the partial scattering functions enables us to determine the radius of gyration of the axial polymer and the one-dimensional array of the cyclic molecules in PR.

\section{SCATTERING THEORY}

In this section, the scattering functions for PR are derived. The backbone of the scattering functions consists of two parts: the Kratky-Porod worm-like chain model ${ }^{18}$ obtained by Yamakawa and Yoshizaki, ${ }^{19}$ and the random copolymer model derived by Endo and Shibayama. ${ }^{20}$ The worm-like chain model is a good model for very stiff polymers, where the correlation between the two bond vectors $\vec{r}_{i}$ and $\vec{r}_{j}$ decays exponentially as

$$
\left\langle\vec{r}_{i} \cdot \vec{r}_{j}\right\rangle=\exp \left(-\frac{l}{l_{\mathrm{p}}}|i-j|\right)
$$

with the length of the bond vector $l$ and the persistence length $l_{\mathrm{p}}$. This model should be applicable to semiflexible PR. The chain thickness of $\mathrm{PR}$, which affects the scattering curve in a high-Q range, is indispensable; however, it can be practically incorporated into the scattering function as described below. It is necessary to consider the three different scattering contributions individually, namely, two self-correlations of the axial polymer and the beaded cyclic molecules and the cross-correlation between the axis and the beads. We will summarize the worm-like chain model and the random copolymer model first, after which the scattering functions for the beads, the axis and the cross-correlation will be derived one by one.

\section{Worm-like chain model}

The worm-like chain model obtained by Yoshizaki et al. ${ }^{19}$ is an empirical equation connecting the Debye function for a Gaussian chain at low $Q$ and the form factor of a rod at high $Q$ in continuity; that is

$$
\begin{aligned}
P_{\mathrm{WC}}\left(Q, L, l_{\mathrm{p}}\right)= & {\left[\left\{1-\chi\left(Q, L, l_{\mathrm{p}}\right)\right\} P_{\text {Debye }}\left(Q, L, l_{\mathrm{p}}\right)\right.} \\
& \left.+\chi\left(Q, L, l_{\mathrm{p}}\right) P_{\text {Rod }}(Q, L)\right] \Gamma\left(Q, L, l_{\mathrm{p}}\right)
\end{aligned}
$$

with the magnitude of the scattering vector $Q(=4 \pi \sin (\theta / 2) / \lambda$; scattering angle $\theta$, wavelength of incident beam $\lambda$ ), contour length $L$ and persistence length $l_{\mathrm{p}}$. The static segment (Kuhn) length $b$ is related to the persistence length by $l_{\mathrm{p}}=b / 2$. The Debye function $P_{\text {Debye }}(Q, L$, $l_{\mathrm{p}}$ ) is defined as

$$
P_{\text {Debye }}\left(Q, L, l_{\mathrm{p}}\right)=\frac{2}{u^{2}}\{\exp (-u)+u-1\}
$$

where $u=R_{\mathrm{g}}^{2} Q^{2}$, with the radius of gyration of the linear polymer $R_{\mathrm{g}}$ given by

$$
R_{\mathrm{g}}^{2}=\frac{L l_{\mathrm{p}}}{3}-l_{\mathrm{p}}^{2}+\frac{2 l_{\mathrm{p}}^{3}}{L}-\frac{2 l_{\mathrm{p}}^{4}}{L^{2}}\left\{1-\exp \left(-\frac{L}{l_{\mathrm{p}}}\right)\right\}
$$

The form factor $P_{\operatorname{Rod}}(Q, L)$ in Equation (2) is written as

$$
P_{\text {Rod }}(Q, L)=\frac{2}{Q L} \operatorname{Si}(Q L)-\left\{\frac{\operatorname{Sin}(Q L / 2)}{Q L / 2}\right\}^{2}
$$

with the sine integral $\operatorname{Si}(x)=\int_{0}^{x} \sin t / t \mathrm{~d} t$. The cross-over region between $P_{\text {Debye }}\left(Q, L, l_{\mathrm{p}}\right)$ and $P_{\text {Rod }}(Q, L)$ in Equation (2) is corrected by functions $\chi\left(Q, L, l_{\mathrm{p}}\right)$ and $\Gamma\left(Q, L, l_{\mathrm{p}}\right)$. The factor $\chi\left(Q, L, l_{\mathrm{p}}\right)$ is given by

$$
\chi\left(Q, L, l_{\mathrm{p}}\right)=\exp \left\{-\left(\frac{\pi R_{\mathrm{g}}^{2} Q}{2 L}\right)^{-5}\right\}
$$

and $\Gamma\left(Q, L, l_{\mathrm{p}}\right)$ is expressed as a function of $Q, L, l_{\mathrm{p}}$ and 35 coefficients calculated by Yoshizaki and Yamakawa. ${ }^{19}$

\section{The random copolymer model}

The random copolymer model of type A-B was derived by Endo et al. on the basis of Gaussian statistics. ${ }^{20}$ In the case of a linear random copolymer with monomer-A and monomer-B, the partial scattering function of monomer-A is described by discretization of the Debye function with interval $l_{0}$ as

$$
\begin{aligned}
P_{\mathrm{RCP}}(Q)= & \frac{1}{N^{2} \Phi_{\mathrm{A}}^{2}} \sum_{i=0}^{n_{0}-1} \sum_{j=0}^{n_{0}-1} A_{m}(i) A_{n}(j) \\
& \times \int_{i l_{0}}^{(i+1) l_{0}} \mathrm{~d} m \int_{j l_{0}}^{(j+1) l_{0}} \mathrm{~d} n \exp \left\{-\frac{Q^{2} b^{2}}{6}|m-n|\right\}
\end{aligned}
$$

where $N$ is the segment number (that is, $N=L / b$ ) and $n_{0}$ is related to the interval $l_{0}$ as $l_{0}=N / n_{0}$. In this definition, $l_{0} \times l_{\mathrm{p}}$ corresponds to the length of a single monomer unit. $A_{m / n}(k)$ is an $n_{0}$-dimensional 
vector defined by

$$
A_{m / n}(k)= \begin{cases}1 & \text { (monomer }- \text { A exists }) \\ 0 & \text { (monomer }- \text { B exists })\end{cases}
$$

for the $k$ th interval. The ensemble average $\left\langle A_{m}(i) \cdot A_{n}(j)\right\rangle$ is

$$
\left\langle A_{m}(i) \cdot A_{n}(j)\right\rangle= \begin{cases}\Phi_{\mathrm{A}} & (\text { for } i=j) \\ \Phi_{\mathrm{A}}^{2} & (\text { for } i \neq j)\end{cases}
$$

with the length fraction of monomer-A component $\Phi_{\mathrm{A}}$.

In the case of a purely random process for the distribution of monomers, $A_{m}(i) \cdot A_{n}(j)$ in Equation (7) can be substituted by $\left\langle A_{m}(i) \cdot A_{n}(j)\right\rangle$; then, an analytic form of Equation (7) is obtained as

$$
\begin{aligned}
P_{\mathrm{RCP}}(Q)= & {\left[\frac{2}{x^{2}} \exp (-x)-1-n_{0} \exp \left(-\frac{x}{n_{0}}\right)+n_{0}\right.} \\
& \left.+\left\{n_{0} \exp \left(-\frac{x}{n_{0}}\right)-n_{0}+x\right\} / \Phi_{A}\right] / N
\end{aligned}
$$

with $x=Q^{2} R_{\mathrm{g}}^{2}$ and $N=1+\left(1-\Phi_{\mathrm{A}}\right) /\left(\Phi_{\mathrm{A}} n_{0}\right)$.

Partial scattering functions for polyrotaxane

The scattering intensity $I(Q)$ of $P R$ in solution is given by

$$
I(Q)=\Delta \rho_{\mathrm{C}}^{2} S_{\mathrm{CC}}(Q)+2 \Delta \rho_{\mathrm{C}} \Delta \rho_{\mathrm{P}} S_{\mathrm{CP}}(Q)+\Delta \rho_{\mathrm{P}}^{2} S_{\mathrm{PP}}(Q)
$$

where $\mathrm{C}$ and $\mathrm{P}$ indicate cyclic molecules and the axial polymer in $\mathrm{PR}$, respectively. $\Delta \rho_{i}(i=\mathrm{C}, \mathrm{P})$ is the scattering contrast between component- $i$ and the solvent, that is, $\Delta \rho_{i}=\rho_{i}-\rho_{\mathrm{S}}$ with the scattering length densities of component $i, \rho_{i}$ and that of the solvent, $\rho_{\mathrm{S}} . S_{i j}(Q)$ denotes the partial scattering function in $P R$, namely, $S_{\mathrm{CC}}(Q)$ and $S_{\mathrm{PP}}(Q)$, which represent the intracorrelation of cyclic molecules and the axial polymer, respectively; $S_{\mathrm{CP}}(Q)$ expresses the intercorrelation (or crosscorrelation) between cyclic molecules and the axial polymer. In Figure 1, a conceptual scheme of the partial scattering functions is illustrated. Derivation of each $S_{i j}(Q)$ will be described below without considering the intermolecular interaction.

The partial scattering function of the axial polymer, $S_{\mathrm{PP}}(Q)$, can be approximated by the worm-like chain model given by Equation (2):

$$
S_{\mathrm{PP}}(Q)=n_{\mathrm{PR}} \cdot V_{P}^{2} \cdot P_{\mathrm{WC}}\left(Q, L, l_{\mathrm{p}}\right)
$$

where $n_{\mathrm{PR}}$ is the number density of $\mathrm{PR}$ and $V_{\mathrm{P}}$ is the volume of the axial polymer.

For the description of the partial scattering function of cyclic molecules, $S_{\mathrm{CC}}(Q)$, we need to integrate the random copolymer model into the worm-like chain model. The unification can be carried out with substitution of Equation (7) for Equation (3); at the same time, the form factor of a rod given by Equation (5) must be replaced by

$$
\begin{aligned}
P_{\text {Cyl }}^{\text {Discr }}(Q)= & \int_{0}^{\pi / 2} P_{\text {Disk }}^{\text {Hollow }}\left(Q, R_{\text {in }}, R_{\text {out }}\right)\left\{\frac{\sin \left\{Q L \cos \theta /\left(2 n_{0}\right)\right\}}{Q L \cos \theta /\left(2 n_{0}\right)}\right\}^{2} \\
& \times \frac{1}{n_{0}^{2} \Phi_{\mathrm{A}}^{2}} \sum_{i=0}^{n_{0}-1} \sum_{j=0}^{n_{0}-1} A_{m}(i) A_{n}(j) \\
& \times \cos \left\{(i-j) Q L \cos \theta / n_{0}\right\} \sin \theta \mathrm{d} \theta
\end{aligned}
$$

See Equations (A6) and (A7) in Appendix A. Finally, $S_{\mathrm{CC}}(Q)$ is given by

$$
\begin{aligned}
S_{\mathrm{CC}}(Q)= & n_{\mathrm{PR}} \cdot V_{\mathrm{C}}^{2} \cdot\left[\left\{1-\chi\left(Q, L, l_{\mathrm{p}}\right)\right\} P_{\mathrm{RCP}}(Q)\right. \\
& \left.+\chi\left(Q, L, l_{\mathrm{p}}\right) P_{\mathrm{Cyl}}^{\text {Discr }}(Q)\right] \Gamma\left(Q, L, l_{\mathrm{p}}\right)
\end{aligned}
$$

where $V_{C}$ is the total volume of the cyclic molecules threaded on the single axial polymer. $\chi\left(Q, L, l_{\mathrm{p}}\right)$ and $\Gamma\left(Q, L, l_{\mathrm{p}}\right)$ in Equation (14) are assumed to be identical to those given by Yoshizaki and Yamakawa. ${ }^{19}$ In the event that the cyclic molecules distribute randomly on the axial polymer, the analytic forms given by Equations (10) and (A8) can be used.

The intercorrelation between cyclic molecules and the axial polymer, $S_{\mathrm{CP}}(Q)$, can also be calculated with the worm-like chain model; that is, $S_{\mathrm{CP}}(Q)$ can be described as

$$
\begin{aligned}
S_{\mathrm{CP}}(Q)= & n_{\mathrm{PR}} \cdot V_{\mathrm{C}} \cdot V_{\mathrm{P}} \cdot\left[\left\{1-\chi\left(Q, L, l_{\mathrm{p}}\right)\right\} P_{\mathrm{RCP}}^{\text {Cross }}(Q)\right. \\
& \left.+\chi\left(Q, L, l_{\mathrm{p}}\right) P_{\mathrm{Cyl}}^{\text {Cross }}(Q)\right] \Gamma\left(Q, L, l_{\mathrm{p}}\right)
\end{aligned}
$$

where

$$
\begin{aligned}
P_{\mathrm{RCP}}^{\mathrm{Cross}}(Q)= & \frac{1}{N^{2} \Phi_{\mathrm{A}}} \sum_{i=0}^{n_{0}-1} \sum_{j=0}^{n_{0}-1} A_{m}(i) \\
& \times \int_{i l_{0}}^{(i+1) l_{0}} \mathrm{~d} m \int_{j l_{0}}^{(j+1) l_{0}} \mathrm{~d} n \exp \left\{-\frac{Q^{2} l_{\mathrm{p}}^{2}}{6}|m-n|\right\}
\end{aligned}
$$

and

$$
\begin{aligned}
P_{\text {Cyl }}^{\text {Cross }}(Q) & =\int_{0}^{\pi / 2} A_{\text {Disk }}^{\text {Hollow }}\left(Q, R_{\text {in }}, R_{\text {out }}\right)\left\{\frac{\sin \left\{Q L \cos \theta /\left(2 n_{0}\right)\right\}}{Q L \cos \theta /\left(2 n_{0}\right)}\right\}^{2} \\
& \times \frac{1}{n_{0}^{2} \Phi_{\mathrm{A}}} \sum_{i=0}^{n_{0}-1} \sum_{j=0}^{n_{0}-1} A_{m}(i) \times \cos \left\{(i-j) Q L \cos \theta / n_{0}\right\} \sin \theta \mathrm{d} \theta
\end{aligned}
$$

with

$$
\begin{aligned}
A_{\text {Disk }}^{\text {Hollow }}\left(Q, R_{\text {in }}, R_{\text {out }}\right)= & \left\{R_{\text {out }}^{2} \frac{J_{1}\left(Q R_{\text {out }} \sin \theta\right)}{Q R_{\text {out }} \sin \theta}\right. \\
& \left.-R_{\text {in }}^{2} \frac{J_{1}\left(Q R_{\text {in }} \sin \theta\right)}{Q R_{\text {in }} \sin \theta}\right\} \\
& \div\left(\frac{2}{R_{\text {out }}^{2}-R_{\text {in }}^{2}}\right)
\end{aligned}
$$

where $A_{n}(j)$ defined by Equation (8) represents the probability density of the axial polymer, that is, $A_{n}(j)=1$ for any $j$. Furthermore, by assuming that cyclic molecules can locate equally at any site in the axial polymer, we can replace $A_{m}(i)$ by its ensemble average $\left\langle A_{m}(i)\right\rangle=\Phi_{\mathrm{A}}$. In this case,

$$
\begin{aligned}
S_{\mathrm{CP}}(Q)= & n_{\mathrm{PR}} \cdot V_{\mathrm{C}} \cdot V_{\mathrm{P}} \cdot \Phi_{\mathrm{A}} \cdot\left[\left\{1-\chi\left(Q, L, l_{\mathrm{p}}\right)\right\} P_{\text {Debye }}\left(Q, L, l_{\mathrm{p}}\right)\right. \\
& \left.+\chi\left(Q, L, l_{\mathrm{p}}\right) P_{\mathrm{CP}}(Q)\right] \Gamma\left(Q, L, l_{\mathrm{p}}\right)
\end{aligned}
$$

is obtained, where $P_{\mathrm{CP}}(Q)$ can be simplified as

$$
P_{\mathrm{CP}}(Q)=\int_{0}^{\pi / 2} A_{\text {Disk }}^{\text {Hollow }}\left(Q, R_{\text {in }}, R_{\text {out }}\right)\left\{\frac{\sin (Q L \cos \theta / 2)}{Q L \cos \theta / 2}\right\}^{2} \sin \theta \mathrm{d} \theta
$$

\section{Distribution of cyclic molecules on the axial polymer}

As discussed in the above section, the partial scattering functions, $S_{\mathrm{CC}}(Q)$ and $S_{\mathrm{CP}}(Q)$, can reflect the row of cyclic molecules on the axial polymer. The distribution of cyclic molecules on the axial polymer can be evaluated by quantitative analyses of $S_{\mathrm{CC}}(Q)$ and $S_{\mathrm{CP}}(Q)$ using Monte Carlo simulations. 
To investigate biased alignments of the cyclic molecules, we define the conditional probability that two cyclic molecules are successively placed as $\phi_{\mathrm{cc}}$ and the conditional probability that two cyclic molecules are not successively placed as $\phi_{\mathrm{c}}$. By defining the total length fraction of the cyclic molecules as $\Phi_{\mathrm{c}}$, we can relate $\Phi_{\mathrm{c}}$ to $\phi_{\mathrm{cc}}$ and $\phi_{\mathrm{c}}$ as follows:

$$
\phi_{\mathrm{c}}=\frac{\Phi_{\mathrm{c}}}{1-\Phi_{\mathrm{c}}}\left(1-\phi_{\mathrm{cc}}\right)
$$

In the model, $\Phi_{\mathrm{c}}>\phi_{\mathrm{cc}}$ indicates a repulsive interaction between cyclic molecules, and $\Phi_{c}<\phi_{c c}$ indicates an attractive interaction between cyclic molecules. The Monte Carlo simulation for $S_{\mathrm{CC}}(Q)$ can be performed by using Equations ( 8$)$ and (14), where $A_{m, n}(k)$ is given by

$$
A_{m, n}(k)=\left\{\begin{array}{ll}
1 & \left(\text { for } \phi_{k} \leqslant \phi_{c c}\right) \\
0 & \left(\text { for } \phi_{k}>\phi_{c c}\right)
\end{array} \text { in case of } A_{m, n}(k-1)=1\right.
$$

and

$$
A_{m, n}(k)=\left\{\begin{array}{ll}
1 & \left(\text { for } \phi_{k} \leqslant \phi_{c c}\right) \\
0 & \left(\text { for } \phi_{k}>\phi_{c c}\right)
\end{array} \text { in case of } A_{m, n}(k-1)=0\right.
$$

where $\phi_{k}$ is a generated random number between 0 and 1 . In this simple model, however, the expected value for $A_{m}(i)$ is constant, independently of $\phi_{\mathrm{cc}}$ and $\phi_{\mathrm{c}}$. In other words, the ensemble value $<A_{m}(i)>$ is always constant for any values of $\phi_{\mathrm{cc}}$ and $\phi_{\mathrm{c}}$ so that $S_{\mathrm{CP}}(Q)$ cannot be affected by the arrangement of the cyclic molecules. Therefore, it is only possible to obtain information about the array of cyclic molecules by analyzing $S_{\mathrm{CC}}(Q)$ with this method.

\section{Interpolymer interaction}

By condensing the concentration of polymers in solution, the polymers gradually start to overlap each other above the overlap concentration $c^{*}$. In the event that the concentration of the polymers is far below $c^{*}$, the static scattering intensities $I(Q)$ can be described with the form factor $P_{\text {poly }}(Q)$ :

$$
I(Q)=I_{0} \times P_{\text {poly }}(Q)
$$

where $I_{0}$ is the forward scattering intensity, which can be calculated as $I_{0}=n \times V^{2} \times \Delta \rho^{2}$ with the number density of the polymer $n$, the volume of the polymer $V$ and the scattering contrast between the polymer and the solvent $\Delta \rho$. If the concentration of the polymer is in the vicinity of $c^{*}$, Equation (24) is invalid because of the effect of interpolymer interactions. The Zimm formula treats the interpolymer interaction with the second virial coefficient $A_{2}$, which is given by

$$
I(Q)=\frac{I_{0}}{1 / P_{\text {poly }}(Q)+2 A_{2} M c}
$$

where $M$ is the molecular weight of the polymer, and $c$ is the massconcentration of the solute. For polymer solutions, $A_{2}$ can be related to the excluded volume $v$ as

$$
A_{2}=\frac{N_{\mathrm{A}} v}{2 m^{2}}
$$

with Avogadro's number $N_{\mathrm{A}}$ and the molar mass of the monomer, $m$.

Equation (25) was originally derived with single contact approximation and an assumption that $2 \mathrm{~A}_{2} \mathrm{Mc}$ is very small compared with unity; ${ }^{21}$ therefore, the applicable concentration should be rather diluted. In fact, the expression given by Equation (25) has been proved to be valid at much higher concentrations theoretically, ${ }^{21}$ and we have also experimentally confirmed its validity in PR solutions. ${ }^{16}$

The Ornstein-Zernike (O-Z) formula is also typically applied for semidilute polymer solutions, where $I(Q) \propto\left(1+\xi^{2} Q^{2}\right)^{-1}$ (with the correlation length $\xi){ }^{22}$ however, it has been experimentally confirmed that the O-Z formula does not fit PR solutions well because of the considerably large persistence length and thickness of PR. ${ }^{16}$ Therefore, Equation (25) will be combined with Equations (12), (14) and (19) to analyze the partial scattering functions quantitatively.

\section{EXPERIMENTAL PROCEDURE}

\section{Materials}

In this study, PR was composed of poly(ethylene glycol) (PEG) as the axial polymer and $\alpha$-cyclodextrin $(\alpha \mathrm{CD})$ as the cyclic molecule. To execute contrast variation experiments, we synthesized two types of PRs, namely, h-PR consisting of hydrogenated poly(ethylene glycol) (h-PEG) and d-PR consisting of fully deuterated poly(ethylene glycol) (d-PEG). Hydrogenated PEG was purchased from Fluka (Buchs, Switzerland), and the deuterated polymer was purchased from Cambridge Isotope Laboratories Inc (Andover, MA, USA); their polydispersity indices were $<1.2 . \alpha \mathrm{CD}$ was purchased from Nihon Shokuhin Kako Co. Ltd (Tokyo, Japan) ( $\alpha$ CD content $>99 \%$ ). The degree of polymerization of both hydrogenated and deuterated PEG was 800 , which corresponds to $3.5 \times 10^{4} \mathrm{~g} \mathrm{~mol}^{-1}$ with hydrogenated PEG conversion. PEG in PR was endcapped with adamantine to prevent the threaded $\alpha \mathrm{CDs}$ from escaping. The average number of $\alpha \mathrm{CDs}$ per chain was determined as 108 by ${ }^{1} \mathrm{H}$ nuclear magnetic resonance analysis, which is equivalent to $\sim 27 \%$ coverage in length. The detailed synthesis procedure is given elsewhere. ${ }^{23}$

\section{Small-angle neutron scattering}

CV-SANS measurements were performed on the SANS-U diffractometer of the Institute for Solid State Physics, the University of Tokyo, installed in the neutron guide hall at the JRR-3 research reactor of the Japan Atomic Energy Agency in Tokai, Japan. ${ }^{24}$ Experiments were carried out at 4- and 1-m sampleto-detector lengths by using neutrons of $7.0 \AA$ wavelength with $\Delta \lambda / \lambda=0.10$ fullwidth at half-maximum, which covered the range of scattering wave numbers $Q$ from $0.01 \AA^{-1}$ to $0.3 \AA^{-1}$. The scattered neutrons were detected by a twodimensional multiwired ${ }^{3} \mathrm{He}$ detector; thereafter, necessary data corrections such as dark counts (electronic noise and so on) subtraction and cell-scattering subtraction were performed. After these corrections, the data sets were normalized to an absolute scale using the incoherent scattering of a polyethylene thin plate as a standard sample, and circular averaging of the two-dimensional data and incoherent scattering subtraction proceeded.

\section{Contrast variation experiments}

Contrast variation experiments were executed by preparing four solvents with mixtures of hydrogenated and deuterated dimethylsulfoxide (h/d-DMSO), which consisted of 85, 90, 95 and 100 volume \% d-DMSO. The corresponding scattering length densities were $4.46 \times 10^{10}, 4.73 \times 10^{10}, 4.99 \times 10^{10}$ and $5.25 \times 10^{10} \mathrm{~cm}^{-2}$, respectively. In addition, two kinds of axial polymers were used, hydrogenated and deuterated PEG, as mentioned above, to improve the reliability. In Figure 2, the scattering length densities of the components are summarized as a function of the volume fraction of d-DMSO in the solvent, $\phi_{\mathrm{d}-\mathrm{DMSO}}$. The scattering length densities were estimated from the chemical structures and mass densities of the substances. Mass densities were measured using a DMA5000 density meter (Anton Paar, Graz, Austria) with sample solutions at $25^{\circ} \mathrm{C}$ : these densities were $1.14 \mathrm{~g} \mathrm{~cm}^{-3}$ for h-PEG, $1.59 \mathrm{~g} \mathrm{~cm}^{-3}$ for $\alpha \mathrm{CD}$ and $1.10 \mathrm{~g} \mathrm{~cm}^{-3}$ for h-DMSO. The densities for deuterated materials were calculated by replacing protons with deuteriums. The evaluated scattering length densities were $6.46 \times 10^{9}$ and $7.14 \times 10^{10} \mathrm{~cm}^{-2}$ for h-PEG and d-PEG, respectively, and $1.97 \times 10^{10} \mathrm{~cm}^{-2}$ for $\alpha \mathrm{CD}$. The volume fraction of PR in all solutions was $8 \%$. The temperature of the samples was regulated to be $25^{\circ} \mathrm{C}$ by a water-circulating thermostat bath with an accuracy of $\pm 0.1^{\circ} \mathrm{C}$.

The decomposition of the intensities into the partial scattering functions can be executed by singular value decomposition for each $Q$ value by knowing each contrast as precisely as possible. The experimental and theoretical details of the method are described elsewhere. ${ }^{25}$

\section{RESULTS AND DISCUSSION}

Figure 3 shows the scattering intensities observed in the contrast variation experiments for the eight samples, the structures of which were identical and scattering length densities of PEG and the solvents 


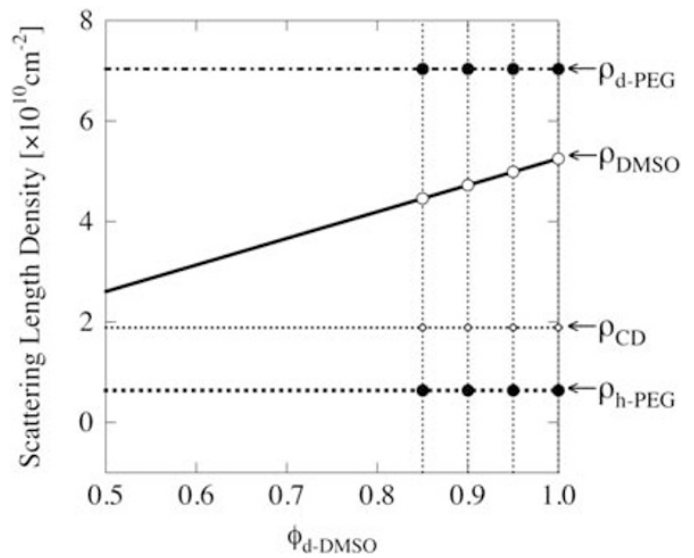

Figure 2 Scattering length densities of dimethylsulfoxide (DMSO), $\rho_{\mathrm{DMSO}}$ deuterated and protonated poly(ethylene glycol) (PEG), $\rho_{\mathrm{d}-\mathrm{PEG}}$ and $\rho_{\mathrm{h}-\mathrm{PEG}}$, respectively; and $\alpha$-cyclodextrin $(\alpha \mathrm{CD}), \rho_{\mathrm{CD}}$, as a function of the volume fraction of deuerated DMSO in the solvent, $\phi_{\mathrm{d}-\mathrm{DMSO}}$. The experimental conditions are marked by filled circles for deuterated and protonated poly(ethylene glycol); open circles for DMSO; and open diamonds for $\alpha$-cyclodextrin.

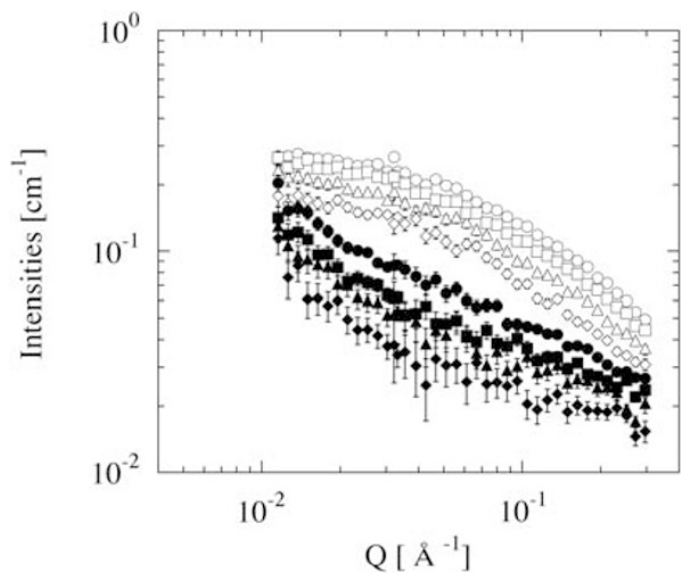

Figure 3 Scattering intensities of the PR solutions for the different contrasts with protonated poly(ethylene glycol) (PEG; open symbols) and deuterated PEG (solid symbols) by tuning the protonated and deuterated dimethylsulfoxide (DMSO) ratio. The curves correspond to $\phi_{\mathrm{d}-\mathrm{DMSO}}=1.00(\bigcirc \bullet)$, $0.95(\square \mathbf{\square}), 0.90(\triangle \mathbf{\Lambda})$ and $0.85(\diamond \diamond)$, where $\phi_{\mathrm{d}-\mathrm{DMSO}}$ is the volume fraction of deuterated DMSO in the solvent.

were different. Obvious characteristics of the curves are upward convexity for the samples with h-PEG and downward convex for samples with d-PEG. This difference is because the sign of the crossterm $\Delta \rho_{\mathrm{c}}$ in Equation (11) is always negative under any experimental conditions, but $\Delta \rho_{p}$ is positive for d-PEG and negative for h-PEG as shown in Figure 2. In Equation (11), therefore, the contrast factor $S_{\mathrm{CP}}(Q)$ becomes positive for h-PEG and negative for d-PEG, which is the origin of the different shapes of the scattering intensities. From this observation, the sign of $S_{\mathrm{CP}}(Q)$ can be estimated to be positive. The average intensity of each curve decreases with the decreasing volume fraction of d-DMSO in the solvent, $\phi_{\mathrm{d}-\mathrm{DMSO}}$, which corresponds to a decrease in the scattering contrast between PR and the solvent with decreasing $\phi_{\mathrm{d}-\text { DMSO. }}$

The obtained partial scattering functions of $\alpha \mathrm{CD}, S_{\mathrm{CC}}(Q), \mathrm{PEG}$, $S_{\mathrm{PP}}(Q)$ and $\alpha \mathrm{CD}$-PEG cross-term, $S_{\mathrm{CP}}(Q)$, are shown in Figure $4 \mathrm{a}$. As mentioned above, $S_{\mathrm{CP}}(Q)$ is positive and the magnitude is between $S_{\mathrm{CC}}(Q)$ and $S_{\mathrm{PP}}(Q)$, and the relation $S_{\mathrm{CC}}(Q)>S_{\mathrm{CP}}(Q)>S_{\mathrm{PP}}(Q)$ is held. The average value of the square root of the ratio of $S_{\mathrm{CC}}(Q)$ to

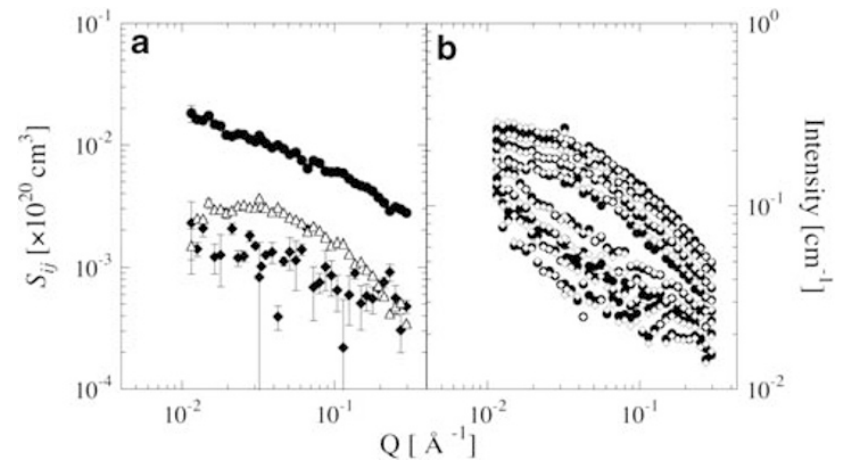

Figure 4 (a) Partial scattering functions obtained of $\alpha$-cyclodextrin $(\alpha C D)$, $S_{\mathrm{CC}}(\bullet), \alpha C D$-poly(ethylene glycol) (PEG) cross-term, $S_{\mathrm{CP}}(\triangle)$, and PEG, $S_{\mathrm{PP}}$ $(\diamond)$. (b) Reconstructed scattering curves $(\diamond)$ compared with experimental values $(\bullet)$. The details of the curves are given in Figure 3.

$S_{\mathrm{PP}}(Q)$, which corresponds to the volume ratio between $\alpha \mathrm{CD}$ and $\mathrm{PEG}$ in $\mathrm{PR}$, is 2.9. The calculated ratio based on the nuclear magnetic resonance result is 2.1 ; thus, these values roughly coincide, and the error may arise from the effect of the structure factor (intermolecular interaction). Furthermore, the $Q$-dependence of $S_{\mathrm{CC}}(Q)$ is similar to that of $S_{\mathrm{PP}}(Q)$, which suggests that $\alpha \mathrm{CD}$ s randomly distribute on the axial chain. The slopes at $\mathrm{Q}>0.1 \AA^{-1}$ are clearly different; that is, the slope of $S_{\mathrm{PP}}(Q)$ is more gradual than those of $S_{\mathrm{CP}}(Q)$ and $S_{\mathrm{CC}}(Q)$. This is because $S_{\mathrm{CP}}(Q)$ and $S_{\mathrm{CC}}(Q)$ reflect the cylinder-like structure of the threaded $\alpha \mathrm{CD}$. These observations are consistent with the structure of PR; that is, $\alpha \mathrm{CDs}$ are mechanically interlocked by PEG. The reconstructed intensities are compared with the experimental values in Figure 4b, where the reconstructed intensities were calculated from the obtained partial scattering functions and the evaluated scattering length densities from Equation (11). The reconstructed intensities reproduce the experimental results quite well, which confirms the good reliability of the overall experimental procedures.

To reveal the detailed structure of PR, the partial scattering functions were analyzed individually. Before the fitting procedure, PR was identified to reduce as many uncertain parameters as possible with the other methods; for example, nuclear magnetic resonance, gel permeation chromatography (GPC), density measurements and checking values in the literature. The fixed parameters are summarized in Table 1.

At first, the persistence length $l_{\mathrm{p}}$ of PR was determined. The best way to determine $l_{\mathrm{p}}$ is by analyzing $S_{\mathrm{PP}}(Q)$, as $S_{\mathrm{PP}}(Q)$ contains only information of the axial part of PR. Unfortunately, the statistical precision of $S_{\mathrm{PP}}(Q)$ is not as good as that of $S_{\mathrm{CP}}(Q)$ and $S_{\mathrm{CC}}(Q)$ because of a lack of contrast variation experiments in which the polymer is mainly visible. Therefore, $S_{\mathrm{CP}}(Q)$ was analyzed with Equation (25), using Equation (19) for the determination of $l_{p}$, which is actually the second-best way. The fitting parameters for $S_{\mathrm{CP}}(Q)$ were $l_{\mathrm{p}}$ and the interaction term $2 \mathrm{~A}_{2} \mathrm{Mc}$ (see Equation (25)), and all of the other parameters could be fixed; therefore, the two parameters could be fitted with high reliability.

The determined $l_{\mathrm{p}}$ from $S_{\mathrm{CP}}(Q)$ is $23 \pm 2 \AA$. This value is rather large compared with that of PEG, which is $11 \AA$ under similar conditions. ${ }^{16}$ This unusually long persistence length is one of the characteristics of PR. ${ }^{13,16}$ The origin of stiffness will be further discussed below. The value was used for the fitting of $S_{\mathrm{PP}}(Q)$ with Equation (12) and $S_{\mathrm{CC}}(Q)$ with Equation (14), using Equation (25) for inclusion of the effect of intermolecular interaction represented by $2 \mathrm{~A}_{2} \mathrm{Mc}$. In Figure 5, the partial scattering functions with the resulting fitting curves are shown. The curves can reproduce the experimental results fairly well. The obtained parameters are summarized in Table 2. 
Table 1 Characteristics of polyrotaxane used in this study

\begin{tabular}{|c|c|}
\hline \multicolumn{2}{|l|}{ PEG } \\
\hline Molecular weight ${ }^{\mathrm{a}}$ & $35000\left(\mathrm{~g} \mathrm{~mol}^{-1}\right)$ \\
\hline Mass density $\left(\text { at } 25^{\circ} \mathrm{C}\right)^{b}$ & $1.14\left(\mathrm{~g} \mathrm{~cm}^{-3}\right)$ \\
\hline Volume (at $25^{\circ} \mathrm{C}$ ), b & $51.00\left(\mathrm{~nm}^{-3}\right)$ \\
\hline Contour lengtha, c & $278(\mathrm{~nm})$ \\
\hline \multicolumn{2}{|l|}{$\alpha C D$} \\
\hline Molecular weight ${ }^{a}$ & $972.84\left(\mathrm{~g} \mathrm{~mol}^{-1}\right)$ \\
\hline Mass density $\left(\text { at } 25^{\circ} \mathrm{C}\right)^{b}$ & $1.59\left(\mathrm{~g} \mathrm{~cm}^{-3}\right)$ \\
\hline Volume (at $\left.25^{\circ} \mathrm{C}\right)^{\mathrm{a}, \mathrm{b}}$ & $1.02\left(\mathrm{~nm}^{-3}\right)$ \\
\hline Number per one PR molecule ${ }^{d}$ & 108 \\
\hline Size (height $\times$ outer radius $\times$ inner radius) ${ }^{c}$ & $0.7 \times 0.72 \times 0.25(\mathrm{~nm})$ \\
\hline
\end{tabular}

PR

Number density of PR in the solution ${ }^{\mathrm{e}}$

$4.89 \times 10^{17}\left(1 \mathrm{~cm}^{-3}\right)$

Abbreviations: $\alpha \mathrm{CD}, \alpha$-cyclodextrin; GPC, gel permeation chromatography; NMR, nuclear magnetic resonance; $\mathrm{PEG}$, poly(ethylene glycol); PR, polyrotaxane.

magnetic resonance; $P E G$, poly(ethylene glycol); $P R$, polyrotaxane.
Characteristics of $P R$ identified by GPC ${ }^{a}$, a density meter ${ }^{b}$, literature values ${ }^{c}, N^{2} R^{d}$ and Characteristics of PR identified by
conditions of sample preparatione.

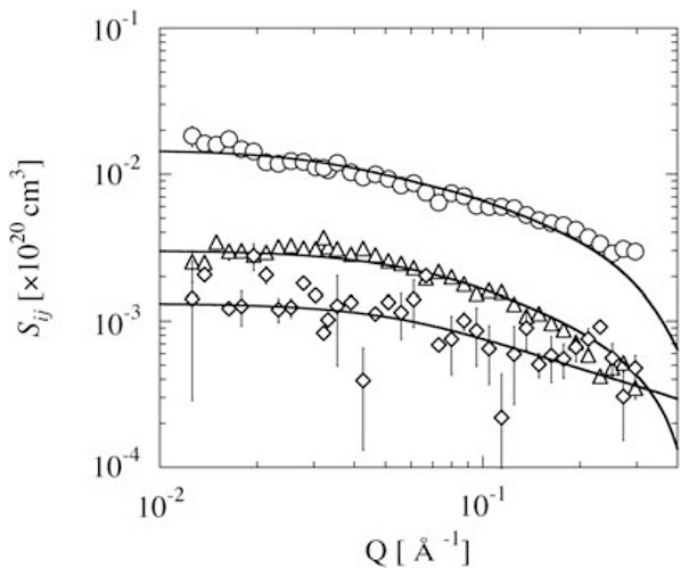

Figure 5 Partial scattering functions of $\alpha$-cyclodextrin $(\alpha C D), S_{C C}(O)$, $\alpha \mathrm{CD}$-poly(ethylene glycol) (PEG) cross-term, $S_{\mathrm{CP}}(\triangle)$, and PEG, $S_{\mathrm{PP}}(\diamond)$. The fitting results are shown by solid curves.

Table 2 Extracted values by analyses of the partial scattering functions

\begin{tabular}{lcccc}
\hline & $\mathrm{I}_{p}\left(A^{\circ}\right)$ & $2 \mathrm{~A}_{2} \mathrm{Mc}$ & $\mathrm{A}_{2}\left(\mathrm{~cm}^{3} \mathrm{molg}^{-2}\right)$ & $v\left(n m^{3}\right)$ \\
\hline$S_{\mathrm{cp}}(Q)$ & $23 \pm 2$ & $90.1 \pm 1$ & $1.5 \times 10^{-2}$ & 2.1 \\
$S_{\mathrm{pp}}(Q)$ & & $95.3 \pm 1$ & $4.8 \times 10^{-2}$ & 0.3 \\
$S_{\mathrm{cc}}(Q)$ & & $39.2 \pm 1$ & $2.2 \times 10^{-3}$ & 6.9
\end{tabular}

Results of fitting with Monte Carlo simulations

\begin{tabular}{ccccc}
\hline & $\phi_{c c}$ & $2 \mathrm{~A}_{2} \mathrm{Mc}$ & $\mathrm{A}_{2}\left(\mathrm{~cm}^{3} \mathrm{molg}^{-2}\right)$ & $v\left(\mathrm{~nm}^{3}\right)$ \\
\hline$S_{\mathrm{cc}}(Q)$ & $0.253 \pm 0.02$ & $40.5 \pm 1$ & $2.3 \times 10^{-3}$ & 7.1 \\
\hline
\end{tabular}

The second virial coefficients, $A_{2}$, of each partial scattering function were separately obtained. For the calculation of $A_{2}$ for $S_{\mathrm{CP}}(Q)$, $\sqrt{M_{\mathrm{PEG}} \times M_{\alpha \mathrm{CD}}}$ and $\sqrt{c_{\mathrm{PEG}} \times c_{\alpha \mathrm{CD}}}$ were applied, where $M_{\mathrm{PEG} / \alpha \mathrm{CD}}$ and $c_{\mathrm{PEG} / \alpha \mathrm{CD}}$ are the molecular weight and mass concentration of PEG/ $\alpha \mathrm{CD}$, respectively. The obtained $A_{2}$ for $S_{\mathrm{CC}}(Q)$ is reasonably comparable to the results in Mayumi et al. ${ }^{16}$ On the other hand, $A_{2}$ values for $S_{\mathrm{CP}}(\mathrm{Q})$ and $S_{\mathrm{PP}}(\mathrm{Q})$ are one order of magnitude larger

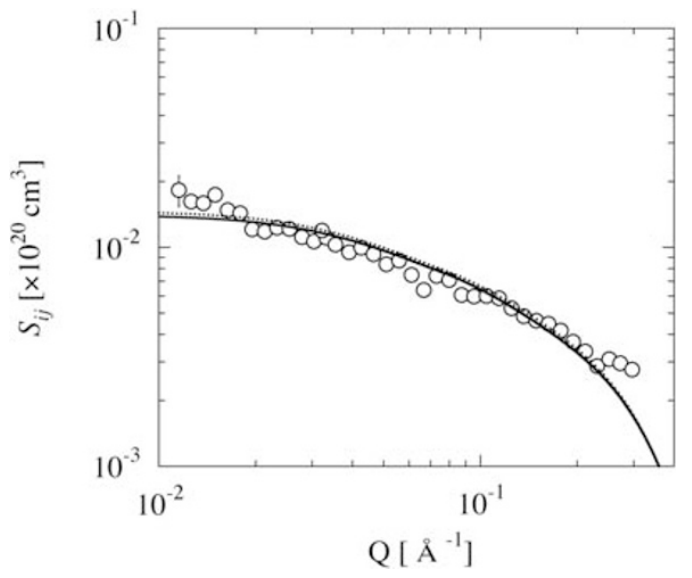

Figure 6 Partial scattering function of $\alpha$-cyclodextrin $(\alpha C D), S_{C C}(O)$. The solid curve is the fitting result obtained by considering the biased arrangement of $\alpha \mathrm{CDs}$ in PR. The dotted line shows the fitting results by assuming a random distribution of $\alpha \mathrm{CDs}$ in $\mathrm{PR}$.

than that of $S_{\mathrm{CC}}(Q)$. The excluded volume $v$ can be related to $A_{2}$ through Equation (26); thus, $A_{2} \propto v$. Therefore, these relatively large $A_{2}$ values compared with those of bare PEG may directly correspond to the large excluded volume of PEG in PR because of the coating of $\alpha \mathrm{CD}$.

All of the above analyses and subsequent discussions were based on the assumption that the distribution of $\alpha \mathrm{CD}$ in $\mathrm{PR}$ is random. In other words, no specific interaction among $\alpha \mathrm{CD}$ s is assumed. Then, consistent results were obtained. To investigate the array of $\alpha \mathrm{CD}$ s on the axial polymer in more detail, the conditional probability that two $\alpha \mathrm{CDs}$ are successively placed, $\phi_{\mathrm{cc}}$, defined by Equations (21)-(23), was evaluated using Monte Carlo simulations, as explained in $\$ 2.5$. In Figure 6, the fitting results are exhibited, together with the fitting curve obtained by assuming a random distribution of $\alpha$ CDs. Sampling was repeated until the relative variation between the $n$th and $(n+1)$ th step became $<10^{-2}$. Otherwise, the value was the average of $10^{3}$ samplings. For the fitting procedure, the persistence length $l_{\mathrm{p}}$ was fixed at $23 \AA$, preliminarily estimated by $S_{\mathrm{CP}}(Q)$ fitting; the evaluated parameters are listed in Table 2. No essential difference for the fitting curves was observed between the results assuming random or biased distribution, and $\phi_{\mathrm{cc}}=0.25$ was achieved, which is quite close to the average length fraction of $\alpha \mathrm{CDs}, \Phi$, which is $\Phi=0.27$. This result also supports the random distribution of $\alpha \mathrm{CDs}$ in PR.

Finally, we discuss briefly the effect of the alignment of the cyclic molecules threaded with the axial polymer on conformation of PR. Let us assume that we have a polymer with contour length $L$ and Kuhn length $b$. In this case, the statistical segment number $N$ may be defined as $N=L / b$. Ideally, the conformational entropy $S_{\mathrm{p}}$ can be given on the basis of Gaussian statistics as

$$
S_{\mathrm{p}}=k_{\mathrm{B}} \ln W_{\mathrm{p}}
$$

where

$$
W_{\mathrm{p}}=\left(\frac{3}{2 \pi N}\right)^{3 / 2} 2^{N+3} \exp \left(-\frac{3 R^{2} N}{2 L^{2}}\right)
$$

with the Boltzmann constant $k_{\mathrm{B}}$ and the length of the end-to-end vector $R$. Next, the effect of arrangement of the cyclic molecules is considered. The maximum number of cyclic molecules in one molecule of PR is defined as $n_{\mathrm{c}}$, and the number fraction of the cyclic molecules in PR is defined as $\phi_{\mathrm{c}}$. By assuming that the cyclic molecules distribute randomly in $\mathrm{PR}$, which is strongly supported by our 


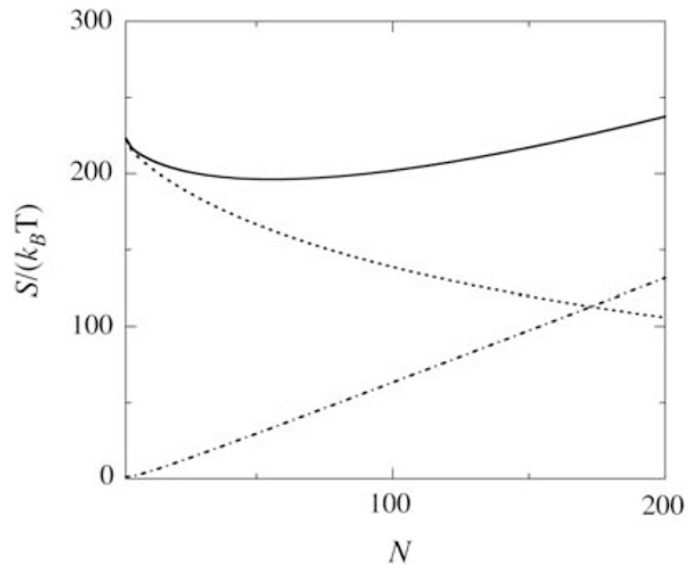

Figure 7 Conformational entropy of PR. Total entropy (solid line) is separated into conformational entropy (dot-dash line) and entropy due to the array of cyclic molecules (dotted line). The fixed parameters are $n_{\mathrm{c}}=400$ and $\phi_{\mathrm{c}}=0.25$ (see text)

analyses of the partial scattering functions, and that the cyclic molecules are prohibited from moving to the next segment, the number of alignments of the cyclic molecules, $W_{\mathfrak{c}}$, is given by

$$
W_{\mathrm{c}}=\left[\frac{\left(n_{\mathrm{c}} / N\right) !}{\left(\phi_{\mathrm{c}} n_{\mathrm{c}} / N\right) !\left\{\left(1-\phi_{\mathrm{c}}\right) n_{\mathrm{c}} / N\right\} !}\right]^{N}
$$

The continuous description of Equation (29) is given by

$$
W_{\mathrm{c}}=\left[\frac{N \cdot \Gamma\left(n_{\mathrm{c}} / N\right)}{n_{\mathrm{c}} \phi_{\mathrm{c}}\left(1-\varphi_{\mathrm{c}}\right) \cdot \Gamma\left(\phi_{\mathrm{c}} n_{\mathrm{c}} / N\right) \cdot \Gamma\left\{\left(1-\phi_{\mathrm{c}}\right) n_{\mathrm{c}} / N\right\}}\right]^{N}
$$

with Gamma function $\Gamma(x)$ and the relation $x !=x \times \Gamma(x)$. The total entropy, $S_{\mathrm{T}}$, is then given by

$$
S_{\mathrm{T}}=S_{\mathrm{p}}+S_{\mathrm{c}}=k_{\mathrm{B}} \ln W_{\mathrm{p}}+k_{\mathrm{B}} \ln W_{\mathrm{c}}
$$

In Figure $7, S_{\mathrm{T}}, S_{\mathrm{p}}$ and $S_{\mathrm{c}}$ are exhibited as functions of $N$ by fixing $n_{\mathrm{c}}=400$ and $\phi_{\mathrm{c}}=0.25$, which are similar to the conditions of PR we used, assuming $R / L$ is very small so that the exponential term in Equation (28) is approximately equal to unity. $S_{\mathrm{p}}$ and $S_{\mathrm{c}}$ have different $N$ dependencies: $S_{\mathrm{p}}$ is a monotonically increasing function of $N$, and $S_{\mathrm{c}}$ is a monotonically decreasing function of $N$. As a result, the total entropy $S_{\mathrm{T}}$ has a minimum. For increasing entropy to reduce the free energy, $N$ may approach 0 , which is a quasi-stable state, or increase beyond the minimum entropy, which is the stable state in this description. A decrease in $N$ corresponds to an enlargement of the persistence length, which is the same as stiffening PR by purely entropic means. In this discussion, the elastic property of the polymer is missing, which normally limits the increase of $N$, so that the entropic effect of the array of cyclic molecules may become important in the case that both $N$ and $\phi_{\mathrm{c}}$ are small. In the event that $\phi_{\mathrm{c}}$ becomes larger, the interaction between cyclic molecules should become considerably important.

\section{CONCLUSIONS}

We investigated the static structure of $\mathrm{PR}$ in a good solvent by means of CV-SANS. The partial scattering functions of $\alpha \mathrm{CD}, S_{\mathrm{CC}}(Q), \mathrm{PEG}$, $S_{\mathrm{CC}}(Q)$ and the cross-term between $\alpha \mathrm{CD}-\mathrm{PEG}, S_{\mathrm{CP}}(Q)$ were extracted by performing contrast variation experiments with high precision. Theoretical equations of each partial scattering function were derived on the basis of the worm-like chain model by assuming a random distribution of $\alpha$ CDs threaded on PEG. This enabled us to evaluate the detailed structural properties of PR in solution, such as persistence length of the axial polymer, alignment of $\alpha \mathrm{CD}$ on the axis and intermolecular interaction with the quantitative comparison between experimental results and the theory. Our results strongly support the idea that $\alpha$ CDs distribute randomly on PEG axial chains. The persistence length of PR determined from $S_{\mathrm{CP}}(Q)$, which was $23 \AA$, and the second virial coefficient for $S_{\mathrm{CC}}(Q)$ were consistent with our former results. ${ }^{16}$ The other two second virial coefficients of PEG and the cross-correlation between $\alpha \mathrm{CD}$ and PEG were also obtained, which were one order of magnitude larger than that of $S_{\mathrm{CC}}(Q)$. We conclude that this is because of the specific structure of PR; that is, PEG is coated randomly by $\alpha \mathrm{CD}$. The origin of the relatively large persistence length of PR was discussed very qualitatively by considering the entropic contribution of the randomly distributing $\alpha \mathrm{CD}$, which behaves in a manner opposite to that of conformational entropy. This entropic effect may be important for PR with a small inclusion of $\alpha \mathrm{CDs}$.

Supramolecules are one of the highly promising candidates for the development of future molecular devices, and this study has proved that CV-SANS is a unique and efficient tool to explore such complex structures consisting of multiple components with nanoscale resolution.

\section{ACKNOWLEDGEMENTS}

This work was partially supported by the Ministry of Education, Science, Sports and Culture, Japan (Grant-in-Aid for Scientific Research on Priority Areas, 2006-2010, No. 18068004 and Grant-in-Aid for Scientific Research (S), 2008-2012, No. 20221005). The SANS experiment was performed with the approval of the Institute for Solid State Physics, The University of Tokyo, at the Japan Atomic Energy Agency, Tokai, Japan (Proposal No. 7607).

1 Yamakawa, H. Modern Theory of Polymer Solutions (Harper and Row, New York, 1971).

2 Doi, M \& Edwards, S. F The Theory of Polymer Dynamics (Oxford University Press, Oxford, 1988).

3 Yamakawa, H. Helical Wormlike Chain in Polymer Solutions (Springer-Verlag, Berlin, 1997).

4 Kuhn, W. Über die Gestalt fadenförmiger Moleküle in Losungen. Kolloid-Z. 68, 2-18 (1934).

5 Flory, P. J. The configuration of real polymer chains. J. Chem. Phys. 17, 303-310 (1949).

6 Yamakawa, H. \& Fujii, M. Statistical mechanics of helical wormlike chains. I. Differential equations and moments. J. Chem. Phys. 64, 5222-5228 (1976).

7 Lehn, J. M. Supramolecular chemistry. Science 260, 1762-1763 (1993).

8 Harada, A., Li, J. \& Kamachi, M. The molecular necklace: a rotaxane containing many threaded $\alpha$-cyclodextrins. Nature 356, 325-327 (1992).

9 Harada, A., Hashidzume, A., Yamaguchi, H. \& Takashima, Y. Polymeric rotaxanes. Chem. Rev. 109, 5974-6023 (2009).

10 Okumura, Y. \& Ito, K. The polyrotaxane gel: a topological gel by figure-of-eight crosslinks. Adv. Mater. 13, 485-487 (2001).

11 Ito, K. Novel cross-linking concept of polymer network: synthesis, structure, and properties of slide-ring gels with freely movable junctions. Polym. J. 39, 489-499 (2007).

12 Karino, T., Okumura, Y., Ito, K. \& Shibayama, M. SANS studies on spatial inhomogeneities of slide-ring gels. Macromolecules 37, 6177-6182 (2004).

13 Fleury, G., Brochon, C., Schlatter, G., Bonnet, G., Lapp, A. \& Hadziioannou, G. Synthesis and characterization of high molecular weight polyrotaxanes: towards the control over a wide range of threaded a-cyclodextrins. Soft Matter 1, 378-385 (2005).

14 Jarroux, N., Guegan, P., Cheradame, H. \& Auvray, L. High conversion synthesis of pyrene end functionalized polyrotaxane based on poly(ethylene oxide) and $\alpha$-cyclodextrins. J. Phys. Chem. B 109, 23816-23822 (2005).

15 Travelet, C., Schlatter, G., Hebraud, P., Brochon, C., Lapp, A., Anokhin, D. V., Ivanov, D. A., Gaillard, C. \& Hadziioannou, G. Multiblock copolymer behaviour of $\alpha$-CD/PEO-based polyrotaxanes: towards nano-cylinder self-organization of $\alpha$-CDs. Soft Matter 4, 18551860 (2008).

16 Mayumi, K., Osaka, N., Endo, H., Yokoyama, H., Sakai, Y., Shibayama, M. \& Ito, K. Concentration-induced conformational change in linear polymer threaded into cyclic molecules. Macromolecules 41, 6480-6485 (2008).

17 Mayumi, K., Endo, H., Osaka, N., Yokoyama, H., Nagao, M., Shibayama, M. \& Ito, K. Mechanically interlocked structure of polyrotaxane investigated by contrast variation small-angle neutron scattering. Macromolecules 42, 6327-6329 (2009).

18 Kratky, O. \& Porod, G. Röntgenuntersuchung gelöster Fadenmoleküle. Rec. Trav. Chim. Pays-Bas. 68, 1106-1123 (1949) 
19 Yoshizaki, T. \& Yamakawa, H. Scattering functions of wormlike and helical wormlike chains. Macromolecules 13, 1518-1525 (1980).

20 Endo, H. \& Shibayama, M. Static partial scattering functions for linear and ring random copolymers. Polym. J. 42, 157-160 (2010).

21 Zimm, B. H. The scattering of light and the radial distribution function of high polymer solutions. J. Chem. Phys. 16, 1093-1099 (1948).

22 Daoud, M., Cotton, P., Farnoux, B., Jannink, G., Sarma, G, Benoit, H., Duplessix, R., Picot, C. \& de Gennes, P. G. Solutions of flexible polymers. Neutron experiments and interpretation. Macromolecules 8, 804-818 (1975).

\section{APPENDIX A}

\section{FORM FACTOR OF A DISCRETE ROD}

The scattering amplitude of a rod of length $L$ is given by

$$
\begin{aligned}
A_{\text {Rod }}(Q) & =\frac{1}{L} \int_{-L / 2}^{L / 2} \exp (-i Q x \cos \theta) \mathrm{d} x \\
& =\frac{2}{Q L \cos \theta} \sin \left(\frac{Q L \cos \theta}{2}\right)
\end{aligned}
$$

where the classic coordinate system with the $z$ axis in the direction of the vector $Q(Q \cdot r=Q r \cos \theta)$ is used. Then, an analytical form of the form factor of the rod can be obtained:

$$
\begin{aligned}
P_{\text {Rod }}(Q) & =\int_{0}^{\pi / 2}\left\{A_{\text {Rod }}(Q)\right\}^{2} \sin \theta \mathrm{d} \theta \\
& =\frac{2}{Q L} \operatorname{Si}(Q L)-\left\{\frac{\sin (Q L / 2)}{Q L / 2}\right\}^{2}
\end{aligned}
$$

which is identical to Equation (5).

We discretize Equation (A1) by assuming that the rod consists of monomer-A and monomer- $\mathrm{B}$, where the total number of monomers is $n_{0}$ and the length of one monomer unit is $L / n_{0}$. The discretization proceeds as follows:

$$
\begin{aligned}
A_{m}^{\mathrm{Rod}}(Q)= & \frac{1}{L} \int_{-L / 2}^{L / 2} \exp (-i Q x \cos \theta) \mathrm{d} x \\
= & \frac{\exp (i Q L \cos \theta / 2)}{L} \int_{0}^{L} \exp (-i Q x \cos \theta) \mathrm{d} x \\
= & \frac{\exp (i Q L \cos \theta / 2)}{L} \sum_{j=0}^{n_{0}-1} \int_{j L / n_{0}}^{(j+1) L / n_{0}} \exp (-i Q x \cos \theta) \mathrm{d} x \\
= & \frac{\exp (i Q L \cos \theta / 2)}{i Q L \cos \theta} \sum_{j=0}^{n_{0}-1}\left[\exp \left(-i \frac{j Q L \cos \theta}{n_{0}}\right)\right. \\
& -\frac{\left.\exp \left\{-i \frac{(j+1) Q L \cos \theta}{n_{0}}\right\}\right]}{i Q L \cos \theta}\left\{1-\exp \left(-i \frac{Q L \cos \theta}{n_{0}}\right)\right\} \\
& \times \sum_{j=0}^{n_{0}-1} \exp \left(-i \frac{j Q \cos \theta}{n_{0}}\right) \\
= & \frac{1}{i} \frac{\sin \left\{Q L \cos \theta /\left(2 n_{0}\right)\right\}}{Q L \cos \theta /\left(2 n_{0}\right)} \exp \left\{\frac{i Q\left(n_{0}-1\right) L \cos \theta}{2 n_{0}}\right\} \\
& \times \frac{1}{n_{0}} \sum_{j=0}^{n_{0}-1} \exp \left(-i \frac{j Q L \cos \theta}{n_{0}}\right)
\end{aligned}
$$

where $P_{\mathrm{R}}(x)=2 \operatorname{Si}(x) / x-\{\sin (\mathrm{x}) / \mathrm{x}\}^{2}$ with the sine integral $\operatorname{Si}(x)$.

In the case of a discrete cylinder with base radius $R$ and length $L$, instead of the discrete rod, Equation (A4) is modified as

$$
\begin{aligned}
P_{\mathrm{Cyl}}^{\text {Discr }}(Q)= & \int_{0}^{\pi / 2} P_{\text {Disk }}(Q R \sin \theta)\left\{\frac{\sin \left\{Q L \cos \theta /\left(2 n_{0}\right)\right\}}{Q L \cos \theta /\left(2 n_{0}\right)}\right\}^{2} \\
& \times \frac{1}{n_{0}^{2}} \sum_{j=0}^{n_{0}-1} \sum_{k=0}^{n_{0}-1} A_{m}(j) A_{n}(k) \\
& \times \cos \left\{(j-k) Q L \cos \theta / n_{0}\right\} \sin \theta \mathrm{d} \theta
\end{aligned}
$$

with the $n_{0}$-dimensional vector $A_{m / n}(k)$ defined by Equation (8). In the case of a purely random process for the distribution of can be substituted by the ensemble average defined by Equation (9); then, an analytic form of Equation (A4) can be derived as

$$
P_{\operatorname{Rod}}^{\text {Discr }}(Q)=\frac{P_{R}\left(Q L / n_{0}\right)+n_{0} \Phi_{\mathrm{A}}\left\{P_{R}(Q L)-\frac{P_{R}\left(Q L / n_{0}\right)}{n_{0}}\right\}}{1+\Phi_{\mathrm{A}}\left(n_{0}-1\right)}
$$


where $P_{\text {Disk }}(x)=\left\{2 J_{1}(x) / x\right\}^{2}$ with the first-order cylindrical Bessel function $J_{1}(x)$. For hollow cylindrical objects such as cyclic molecules, $P_{\text {Disk }}(x)$ can be substituted by

$$
\begin{aligned}
P_{\text {Disk }}^{\text {Hollow }}\left(Q, R_{\text {in }}, R_{\text {out }}\right)= & \left\{R_{\text {out }}^{2} \frac{J_{1}\left(Q R_{\text {out }} \sin \theta\right)}{Q R_{\text {out }} \sin \theta}\right. \\
& \left.-R_{\text {in }}^{2} \frac{J_{1}\left(Q R_{\text {in }} \sin \theta\right)}{Q R_{\text {in }} \sin \theta}\right\}^{2} \\
& \times\left(\frac{2}{R_{\text {out }}^{2}-R_{\text {in }}^{2}}\right)^{2}
\end{aligned}
$$

where $R_{\mathrm{in}}$ is the internal radius and $R_{\text {out }}$ is the external radius. For the purely random process of the distribution of monomer-A, Equation (A6) can be reduced to

$$
\begin{aligned}
P_{\mathrm{Cyl}}^{\text {Discr }}(Q)= & \int_{0}^{\pi / 2} P_{\text {Disk }}(Q R \sin \theta)\left\{\frac{\sin \left\{Q L \cos \theta /\left(2 n_{0}\right)\right\}}{Q L \cos \theta /\left(2 n_{0}\right)}\right\}^{2} \\
& \times\left[\Phi_{\mathrm{A}}+\frac{\Phi_{\mathrm{A}}^{2}}{n_{0}^{2}}\left\{\frac{\sin (Q L \cos \theta / 2)}{\sin \left\{Q L \cos \theta /\left(2 n_{0}\right)\right\}}\right\}-\Phi_{\mathrm{A}}^{2}\right] \\
& \times \sin \theta \mathrm{d} \theta
\end{aligned}
$$

